\title{
Impact modification of poly(caprolactam) by copolymerization with a low molecular weight polybutadiene
}

\author{
R. J. M. Borggreve* and R. J. Gaymans \\ University of Twente, Department of Chemical Technology, PO Box 217, 7500AE \\ Enschede, The Netherlands
}

(Received 2 October 1987; revised 4 December 1987; accepted 3 February 1988)

\begin{abstract}
Caprolactam and a reactive, low molecular weight polybutadiene were polymerized in an autoclave, followed by post-condensation in the solid state. The rubber concentration was varied $(0-30 \mathrm{wt} \%)$. The morphology of the reaction products was studied by transmission electron microscopy. In the materials with 10 and $20 \mathrm{wt} \%$ polybutadiene, large spherical polybutadiene-rich domains with nylon sub-inclusions were observed. The $30 \mathrm{wt} \%$ polybutadiene product was shown to have a co-continuous structure. The moduli of the materials decrease rapidly with rubber content and only the material with the highest polybutadiene content had a high notched Izod impact strength.
\end{abstract}

(Keywords: nylon-rubber blend; polyamide-polybutadiene blockcopolymer; morphology; rubber concentration; modulus; impact toughness; deformation mechanism)

\section{INTRODUCTION}

The impact toughness of nylons can be improved by plasticizing with water ${ }^{1,2,3}$ or by blending with an elastomer ${ }^{4-8}$. The toughening effect of water in nylon-6 is the result of a lowering of the yield stress of the polymer ${ }^{3}$. At a sufficiently low yield stress plastic deformation is allowed to take place extensively in the nylon matrix, absorbing a large amount of impact energy. Unfortunately, since yield stress and modulus are related $^{9}$, this toughening effect is coupled with a considerable loss in modulus. On the other hand, polyamides can be modified by the incorporation of an elastomeric phase into the matrix by reaction blending. If the size of the dispersed rubber phase is small (of the order of $0.3 \mu \mathrm{m})$ a low rubber content (10-25 vol \%) is sufficient for excellent impact properties, even at low temperature, without a considerable loss in stiffness ${ }^{4}$. The rubber particles are apparently able to induce excessive plastic deformation in the nylon matrix although the overall yield stress of the blend is still relatively high. It is not clear if the combination of high modulus and high impact strength can be achieved with polyamide-rubber block copolymers.

Illing ${ }^{10}$ found that the shock resistance, measured with a falling dart test, of a block copolymer based on $92 \mathrm{wt} \%$ caprolactam and $8 \mathrm{wt} \%$ ethylene-acrylic acid was ten times the shock resistance of pure nylon-6. However, no information was given about the modulus of the copolymer.

Van der Loos et al. ${ }^{11}$ studied ABA block copolyamides of which block $A$ is polycaprolactam and block B polypropylene glycol. The block copolymers were synthesized in a reaction injection moulding machine. When the concentration of the soft phase $B$ exceeded $20 \mathrm{wt} \%$ the impact toughness increased by more than a factor ten. However, the modulus dropped to less than a

* Present address: DSM Research, PO Box 18,6160 MD Geleen, The Netherlands third of the modulus of nylon- 6 . The authors postulate that part of the nylon could be encapsulated by the rubber phase.

For a two-phase system the modulus can be calculated by equations based on theories of Takayanagi ${ }^{2,13}$, Van der Poel ${ }^{14}$ or Kerner ${ }^{15}$. Dickie ${ }^{16}$ and Bohn ${ }^{17}$ have shown that these three theories agree well if applied to a rigid matrix with soft inclusions.

According to Kerner the modulus of polymer-polymer composites can be calculated from the following equation:

$$
\frac{G}{G_{\mathrm{m}}}=\frac{(1-\phi) G_{\mathrm{m}}+(\alpha+\phi) G_{\mathrm{i}}}{(1+\alpha \phi) G_{\mathrm{m}}+\alpha(1-\phi) G_{\mathrm{i}}}
$$

where $G$ is the elastic shear modulus, $\phi$ is the volume fraction of inclusions, subscript $m$ denotes a matrix property, subscript $\mathrm{i}$ denotes an inclusion property and $\alpha$ is a function of $v_{\mathrm{m}}$, the Poisson ratio of the matrix:

$$
\alpha=2\left(4-5 v_{\mathrm{m}}\right) /\left(7-5 v_{\mathrm{m}}\right)
$$

Greco et al. ${ }^{18}$ reported on the polymerization of caprolactam in the presence of a functionalized ethylenepropylene elastomer. Typical nylon-rubber blends were obtained with graft copolymers at the interface. The rubber produced a moderate effect on the impact toughness because of the relatively large rubber particle size. The relatively high viscous rubber apparently could not be dispersed sufficiently finely in the nylon during the reaction in the autoclave.

In this work caprolactam, a low molecular weight polybutadiene-acrylonitril rubber with amine endgroups and a small amount of adipic acid were polymerized. The rubber was chosen because it contains unsaturated bonds, so that it could easily be stained with $\mathrm{OsO}_{4}$ for a morphology study. A block copolymer can be formed when the rubber reacts with the nylon (Scheme 1). The polyamide chains in the copolymer form the hard segments, whereas the polybutadiene block is soft. 


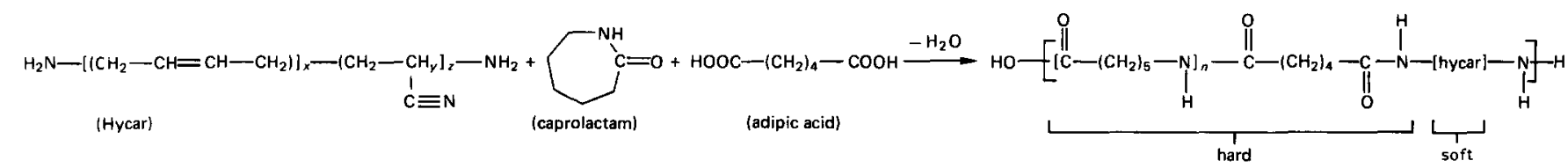

Scheme 1

Table 1 Compositions of reaction mixtures ${ }^{a}$

\begin{tabular}{llll}
\hline & $\begin{array}{l}\text { Hycar } \\
(\text { wt \%) }\end{array}$ & $\begin{array}{l}\mathrm{CL} \\
(\mathrm{wt} \%)\end{array}$ & $\begin{array}{l}\text { Adipic acid } \\
(\mathrm{wt} \%)\end{array}$ \\
\hline Cop10 & 10 & 89.4 & 0.6 \\
Cop20 & 20 & 78.8 & 1.2 \\
Cop30 & 30 & 68.2 & 1.8 \\
\hline
\end{tabular}

${ }^{a}$ To each mixture $5 \mathrm{wt} \% \mathrm{H}_{2} \mathrm{O}$ was added

Because of the great difference in polarity of polyamide and polybutadiene a good phase separation with only a little mixing of phases at the phase boundary was expected. The effect of the composition on the morphology of the two-phase materials was examined with transmission electron microscopy. The moduli and impact behaviour of the materials were evaluated and interpreted in relation to their composition and morphology.

\section{EXPERIMENTAL}

\section{Materials}

Nylon-6 (PA6) was supplied by AKZO (Akulon M224). It had a relative viscosity of 2.5 in $96 \% \mathrm{H}_{2} \mathrm{SO}_{4}$ $\left(1 \mathrm{~g} / 100 \mathrm{ml}, 25^{\circ} \mathrm{C}\right)$. Caprolactam was supplied by DSM. The liquid polybutadiene-acrylonitril was Hycar $1300 \times 21$, supplied by Goodrich BF. Hycar was provided with amine endgroups, $\left[\mathrm{NH}_{2}\right]=0.85 \mathrm{meq} \mathrm{g}^{-1}, M_{\mathrm{n}}=$ $2300 \mathrm{~g} \mathrm{~mol}^{-1}$. Hycar is brown. Adipic acid was obtained from Merck. All components were used without further purification.

\section{Synthesis}

Caprolactam (CL), polybutadiene and adipic acid were polymerized by a two-step procedure:

1 Prepolymerization in the melt in a stirred autoclave. A small amount of water (about $5 \mathrm{wt} \%$ of the total reaction mixture) was used for the ring opening reaction $\left(240^{\circ} \mathrm{C}, 15\right.$ bar, $\left.1 \mathrm{~h}\right)$.

2 Post-condensation reaction in the solid state $\left(170^{\circ} \mathrm{C}\right.$, 1 bar $\mathrm{N}_{2}, 24$ h).

The compositions of the reaction mixtures are given in Table 1 .

Melt flow index

The melt flow index was measured according to ASTM D 1238-65 $\mathrm{T}$ with a Vaschetti and Grosso melt flow apparatus. The test temperature was $235^{\circ} \mathrm{C}$; a $5 \mathrm{~kg}$ block was used.

D.s.c.

The melting behaviour of the materials was studied with a Perkin-Elmer DSC2. Each sample was heated from the granular state to $245^{\circ} \mathrm{C}$, cooled to $100^{\circ} \mathrm{C}$ and reheated. The rate of temperature change was $20^{\circ} \mathrm{C} \mathrm{min}{ }^{-1}$ in each case. The thermogram obtained on second melting was evaluated to determine the melting enthalpy, $\Delta H_{\mathrm{m}}$, and the melting temperature, $T_{\mathrm{m}}$ (the peak of the thermogram). Since the melting enthalpy of $100 \%$ crystalline polyamide- $6, \Delta H_{\mathrm{m}}^{\circ}$, is $230 \mathrm{~J} \mathrm{~g}^{-1}$ (reference 19), the crystallinity fraction $x_{\mathrm{c}}$ of the polyamide phase can be calculated as follows:

$$
x_{\mathrm{c}}=\frac{\Delta H_{\mathrm{m}}}{\Delta H_{\mathrm{m}}^{\mathrm{O}}(1-\phi)} \times 100 \% .
$$

\section{Injection and compression moulding}

The polymers were injection moulded on an Arburg Allrounder 221-35-250 to prepare samples for the Izod test, the three-point bending test and the torsion pendulum test. Compression moulding on a Lauffer OPS40 (10 bar, $230^{\circ} \mathrm{C}$ ) was used to prepare samples for the tensile test.

\section{Infrared spectroscopy}

Thin films were pressed for infrared spectroscopy with the compression moulding machine. Spectra of the materials were taken with a Perkin-Elmer 1310 infrared spectrophotometer.

\section{Transmission electron microscopy}

Thin $(\approx 100 \mathrm{~nm})$ sections of injection moulded bars were obtained by means of cryo-ultramicrotomy. Two microtomes were used: the LKB Cryo-Nova and the Reichert-Jung Ultracut E. Sectioning temperatures varied within the range -125 to $-90^{\circ} \mathrm{C}$. The polybutadiene phase was stained on sections deposited on microscope grids. These grids were kept in the vapour of an aqueous $2 \%$ solution of $\mathrm{OsO}_{4}$ for $30 \mathrm{~min}$ at most. $\mathrm{A}$ Jeol 200 CS transmission electron microscope operated at $200 \mathrm{kV}$ was used to examine the samples.

\section{Materials testing}

All samples were dried before each test $\left(100^{\circ} \mathrm{C}, 24 \mathrm{~h}\right)$, unless otherwise stated. Tensile tests were performed on an Instron machine with a drawing speed of $250 \% \mathrm{~min}^{-1}$ at room temperature (DIN 53455, specimen type 5). The flexural modulus was measured with the Instron machine according to ASTM D790. Shear, elastic and loss moduli were measured with a Myrenne torsion pendulum ATM3 at a frequency of $1 \mathrm{~Hz}$ and a heating rate of $0.5^{\circ} \mathrm{C} \mathrm{min}-1$. Notched Izod impact strength was measured (ISO 180/1A) with a Zwick apparatus.

\section{RESULTS}

Synthesis

The products from the synthesis were yellowish brown. Determining the molecular weight posed a problem since a suitable solvent system for the reaction products was not found. This could be a consequence of the polarity 
Impact modification of poly(caprolactam): R. J. M. Borggreve and R. J. Gaymans

Table 2 Characterization data of nylon-6 and the reaction products

\begin{tabular}{|c|c|c|c|c|c|c|c|}
\hline & $\eta_{\text {rel }}$ & $\begin{array}{l}\text { MFI } 235 / 5 \\
(\mathrm{~g} / 10 \mathrm{~min})\end{array}$ & $\begin{array}{l}T_{\mathrm{m}} \\
\left({ }^{\circ} \mathrm{C}\right)\end{array}$ & $\begin{array}{l}\Delta H_{\mathrm{m}} \\
\left(\mathrm{J} \mathrm{g}^{-1}\right)\end{array}$ & $\begin{array}{l}x_{c} \\
(\%)\end{array}$ & $\begin{array}{l}T_{\mathrm{g}, \mathrm{PA}} \\
\left({ }^{\circ} \mathrm{C}\right)\end{array}$ & $\begin{array}{l}T_{\mathrm{g}, \text { rubber }} \\
\left({ }^{\circ} \mathrm{C}\right)\end{array}$ \\
\hline PA6 & 2.4 & 11.2 & 222 & 62.1 & 27.0 & 64 & - \\
\hline Cop 10 & - & 9.8 & 215 & 44.0 & 21.3 & 52 & -58 \\
\hline Cop20 & - & 8.4 & 214 & 32.5 & 19.7 & 54 & -62 \\
\hline Cop30 & - & - & 214 & 32.8 & 20.4 & 54 & -63 \\
\hline
\end{tabular}
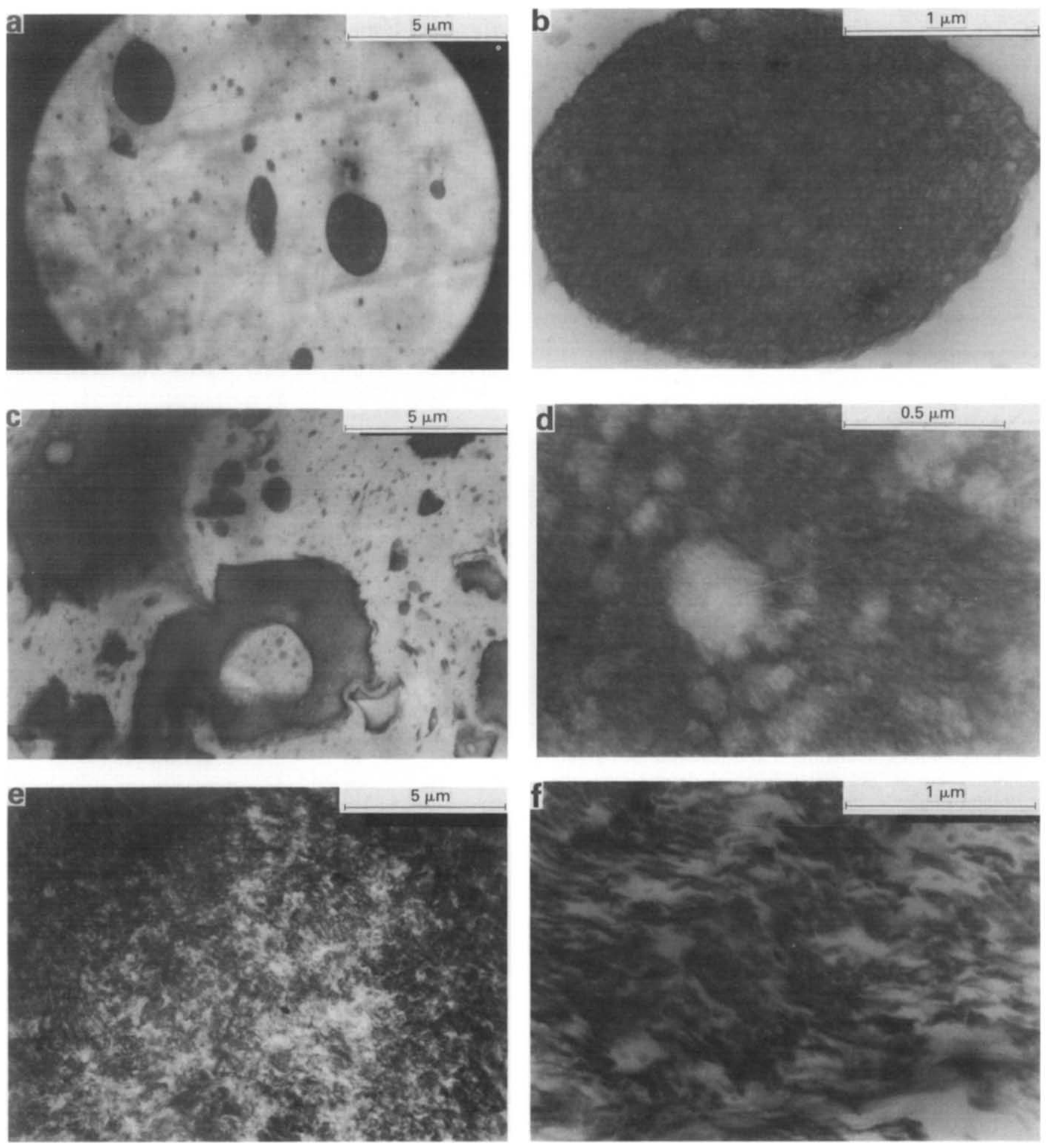

Figure 1 Transmission electron micrographs of polyamide-polybutadiene two-phase materials. The dark areas are the polybutadiene phase, which is stained with $\mathrm{OsO}_{4}$. (a), (b) $\mathrm{Cop} 10$; (c), (d) cop20; (e), (f) cop30. (b) and (d) are magnifications of the polybutadiene-rich phase 

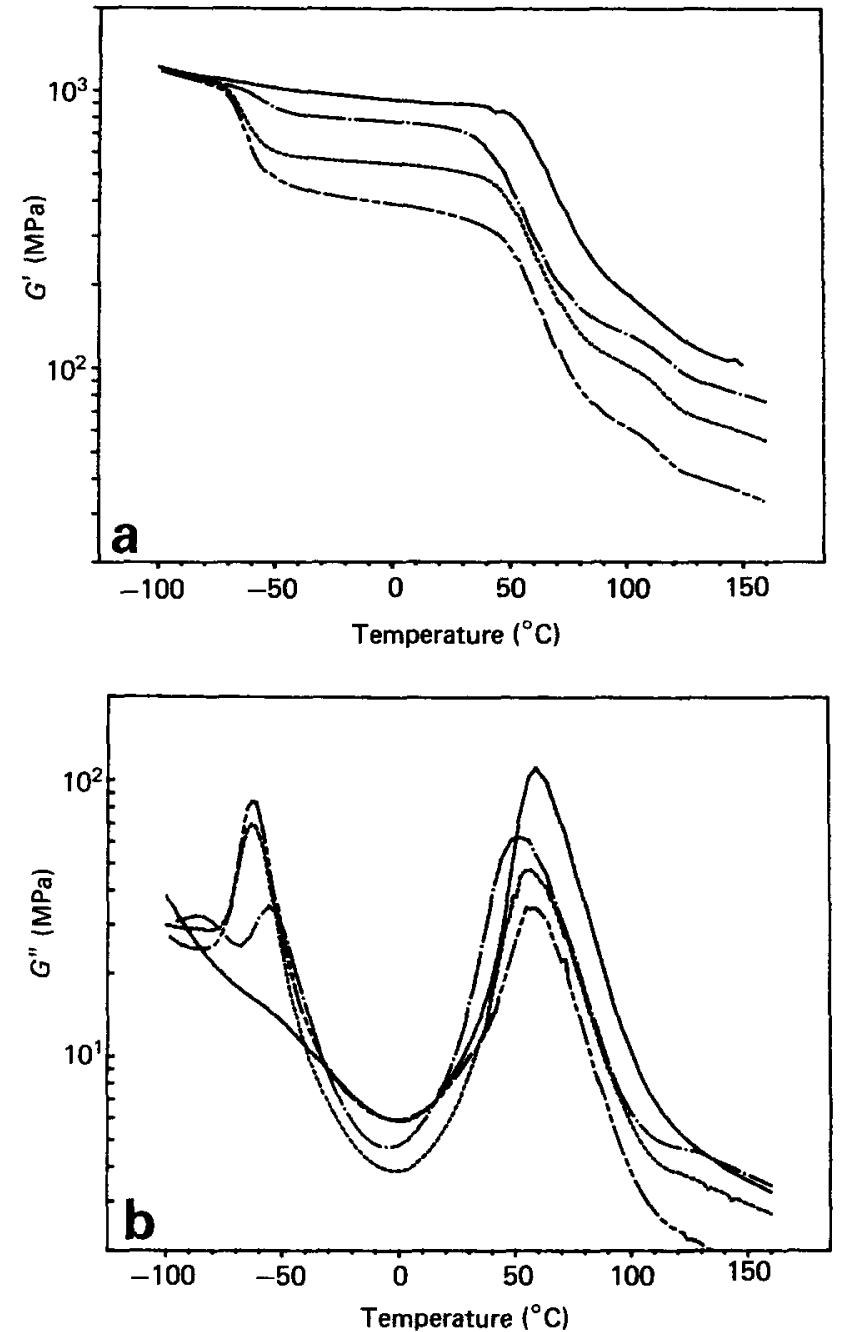

Figure 2 (a) Shear modulus $G^{\prime}$ and (b) loss modulus $G^{\prime \prime}$ as functions of temperature: - - nylon $-6 ;-. \cdots \cdot$, cop $10 ;---$, cop 20 ; - - $\operatorname{cop} 30$

difference between polyamide and polybutadiene. In $m$ cresol, a typical solvent for nylon-6, the reaction products formed swollen systems. This suggests that at least some block copolymer had been formed. This could be verified by infrared spectroscopy. The carbonyl stretching peak at $1700 \mathrm{~cm}^{-1}$ of adipic acid and the $\mathrm{N}-\mathrm{H}_{2}$ stretching peak at $3500 \mathrm{~cm}^{-1}$ of Hycar disappeared in the spectrum of the reaction products, indicating that a reaction took place.

Since there is no direct way of determining the molecular weight we measured the melt flow index (MFI). The MFI and other characterization data of nylon- 6 and the reaction products are given in Table 2 .

The MFI of cop10 and cop20 are lower than the MFI of Akulon M224. The MFI of cop30 could not be registered because of poor flow properties. The MFI results suggest that the molar masses of cop 10, cop20 and cop30 exceed that of Akulon M224. It is known ${ }^{20}$ that the molar mass of nylon-6 only moderately affects the impact strength.

The melting temperatures of the reaction products are lower than the melting temperature of nylon-6. This could be a result of the decrease in lamella thickness of the nylon crystallites as the nylon-block length decreases with increasing rubber content.

The melting enthalpy decreases with decreasing polyamide content. However, cop $10, \operatorname{cop} 20$ and cop30 all have crystallinity fractions $x_{c}$ about $30 \%$ lower than that of neat polyamide- 6 .

\section{Morphology}

The morphology of the reaction products was studied by transmission electron microscopy (Figure 1). The dark regions are due to the $\mathrm{OsO}_{4}$ staining on the polybutadiene. The structure of both cop 10 and cop 20 is inhomogeneous on a micrometre scale. Cop 10 has a dispersion-like morphology. Spherical domains with diameters up to $3 \mu \mathrm{m}$ are formed, which are rich in polybutadiene. Polyamide sub-inclusions seem to be embedded in these particles. The micrograph of cop20 shows both spherical and enlarged polybutadiene-rich domains tending to a continuous network, encapsulating parts of the nylon phase. The high magnification of the polybutadiene-rich phase shows a typical block copolymer layer structure with some polyamide occlusions.

It is not certain whether polybutadiene is present in the matrix of cop 10 and cop20. However, the morphologies of both materials suggest that no pure block copolymers are formed, but rather blends of polyamide- 6 and polyamide-polybutadiene block copolymers. From studies on blends of polystyrene and polystyrenepolybutadiene block copolymers, it is known that the homopolymer will be encapsulated by the domains of the polystyrene blocks if the molecular weight of the block exceeds the molecular weight of the homopolymer ${ }^{21,22}$. Figure 1 suggests that a similar phenomenon has occurred in cop10 and cop20. Owing to the incompatibility of polyamide and polybutadiene, a phase separation occurs shortly after the beginning of the polymerization, leading to typical oil-in-oil emulsions.

The structure of cop30, on the other hand, is more homogeneous on a micrometre scale, although it looks as though some neat nylon is occluded here too. The morphology seems to be co-continuous: both the rubber and the nylon phase form a continuous network.

\section{Mechanical properties}

Figure 2 gives plots of shear and loss modulus against temperature. The glass transition temperatures of both the polyamide phase and the rubber phase change little with composition (Table 2). This suggests a good phase separation. However, the drop in modulus at the lower glass transition temperature is large, especially for cop 30 .

In Figure 3 the normalized shear modulus of the

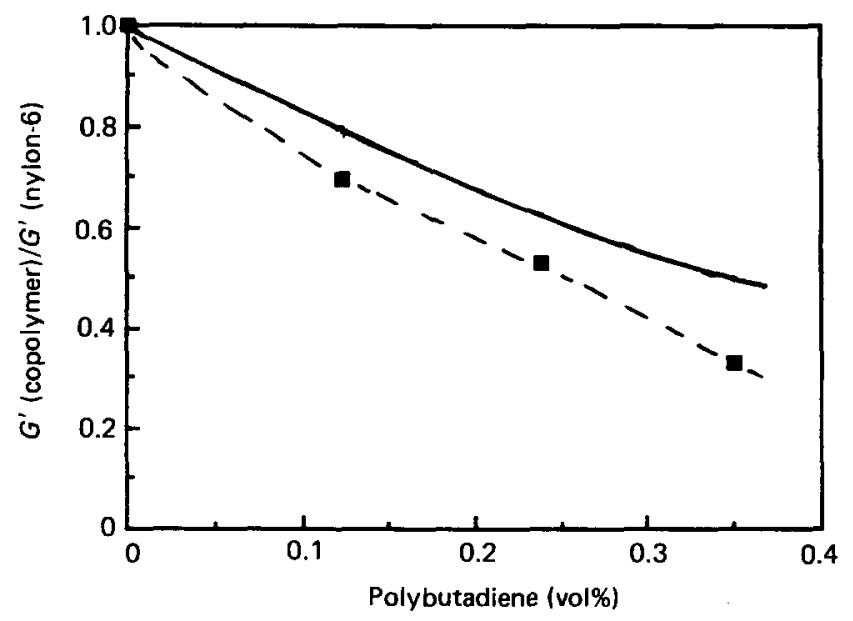

Figure 3 Normalized shear modulus measured at room temperature versus volume fraction polybutadiene. The solid line is calculated from the Kerner equation (equation (1)) 


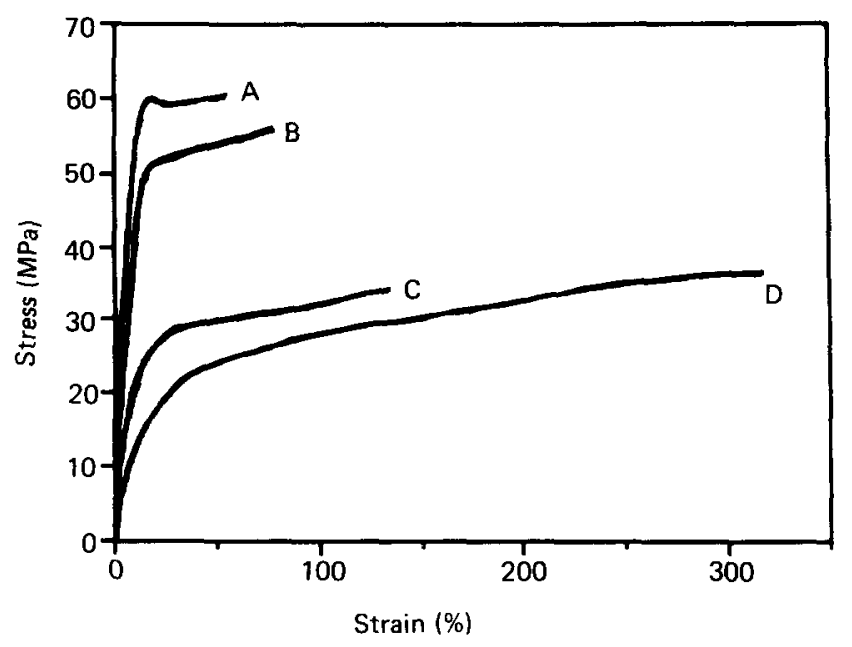

Figure 4 Stress-strain behaviour of the polyamide-polybutadiene two-phase materials: A, nylon-6; B, cop $10 ;$ C, cop20; D, cop30

products at room temperature is plotted against rubber content. The moduli are lower than expected from equation (1). From the electron micrographs it is clear that cop 10 and cop20 contain a dispersed phase rich in polybutadiene, but with some nylon blocks and some occluded nylon polymer. The effective volume fraction of inclusions is therefore higher than the volume fraction of polybutadiene. The part of the polyamide phase which does not practically contribute to the stiffness of the material will be even higher in cop30.

In Figure 4 the stress-strain behaviour of the materials is compared with nylon-6. Young's modulus and yield stress decrease rapidly with increasing rubber content, whereas elongation at break increases.

The notched Izod impact strength was measured over a wide range of temperatures (Figure 5). There is a dramatic difference between the impact behaviour of cop 10 and cop20 and that of cop30. Cop10 and cop20 have impact strengths only twice that of nylon-6, even at high temperatures. On the other hand, cop30 has a high impact strength over the whole temperature range.

On the fracture surfaces of cop 10 and cop20 as well as of cop30 no stress whitening was observed.

\section{DISCUSSION AND CONCLUSIONS}

Cop 10 and cop 20 can be regarded as blends of nylon- 6 and rubbery particles. The small improvement in the impact toughness of these materials can be explained by the large size of the dispersed phase. It has been reported ${ }^{4}$ that the average rubber particle size in nylon-rubber blends must be below $1 \mu \mathrm{m}$ to improve impact toughness significantly. On the other hand, the impact strength of cop30, which seems to be a block copolymer with some nylon occlusions, is excellent although its modulus is low.

A remarkable difference between the tough cop30 and tough nylon-rubber blends can be noticed by studying the fracture surfaces of the two types of material. Whereas the brittle and tough fracture surfaces of nylonrubber blends show intensive stress whitening at the notch tip and over the whole crack path, respectively ${ }^{4}$, the fracture surfaces of cop30 show no stress whitening at all. So, there is strong evidence that different deformation mechanisms take place in nylon-rubber blends and cop30.

The impact toughness of polyamides can be improved by two mechanisms:

1 Lowering of the macroscopic yield stress. This can be done by raising the temperature above the $T_{\mathrm{g}}$ of the nylon, adding a plasticizer like water ${ }^{2,3}$ or adding an elastomer by either blending or copolymerization.

2 A typical rubber dispersion induced toughening mechanism. This is only observed when the average distance between the rubber particles is sufficiently small, as has been demonstrated for nylon-6/EPDM rubber blends ${ }^{4}$. For blends with a low rubber concentration $(\phi<25 \mathrm{vol} \%)$ a small interparticle distance can only be obtained when small particles are used ${ }^{6}$. In the blends, delamination or cavitation of the rubber particles during impact creates voids ahead of the crack tip and when the interparticle distance is small enough, local plane stress states, even in the bulk of the specimen, will generate extensive shear yielding resulting in a tough fracture. The voiding process causes the stress whitening. This mechanism is discussed in more detail elsewhere ${ }^{23}$.

The difference between the two toughening mechanisms is illustrated by Figure 6. Notched Izod impact energies of modified polyamides are given as a function of the normalized flexural modulus, both properties measured at room temperature.

It turns out that the impact strengths of the wetted nylon-6, the nylon-rubber blends with a large rubber particle size ${ }^{4}$, the polyamide-polypropyleneglycol (PPG) block copolymers ${ }^{11}$ and the polyamide-polybutadiene copolymer blends only increase significantly when the modulus is below a certain critical value. That can be achieved at high water or high rubber concentrations. Brown ${ }^{9}$ demonstrated that a direct relation exists between the modulus and the yield stress of a polymeric material. Figure 6 then in fact shows that the four materials are only tough at room temperature when they have a yield stress $\left(\sigma_{y}\right)$ below a critical value $\left(\sigma_{y \text {-crit }}\right)$. For these modified polyamides toughening mechanism 1 obviously applies.

The blends of nylon-6 and EPDM rubber with small

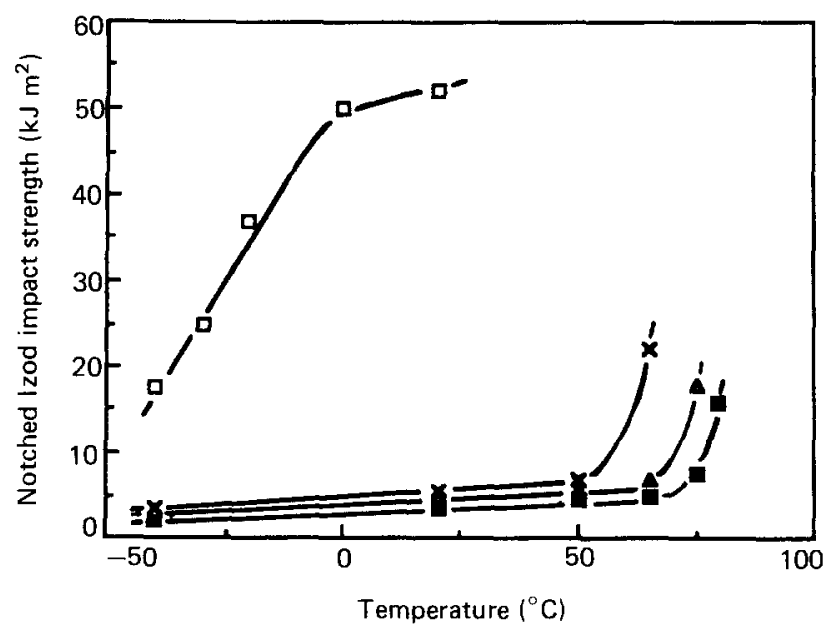

Figure 5 Notched Izod impact strength versus temperature: $\square$, nylon$6 ; \triangle, \operatorname{cop} 10 ; \times, \operatorname{cop} 20 ; \square, \operatorname{cop} 30$ 


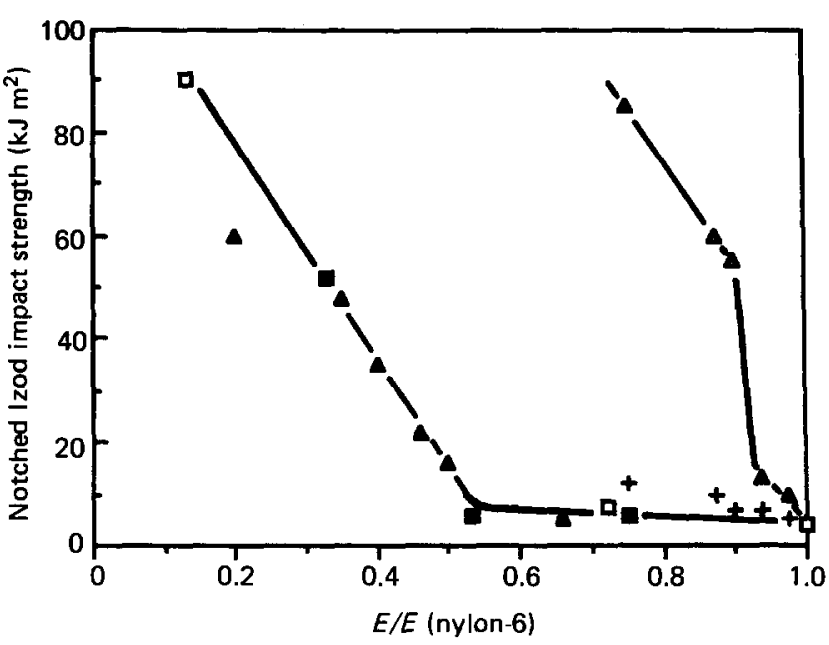

Figure 6 Notched Izod impact strength versus normalized flexural modulus, both measured at $23^{\circ} \mathrm{C}$ : $\square$, cop 10 , cop20, cop30; $\square$, nylon-6 with various water contents $(0,2.3,7.7,11.7 \mathrm{wt} \%)$, after Reference $3 ; \boldsymbol{\Delta}$, nylon-6/PPG block copolymers with various PPG contents $(18,20,22$, $24,26,30 \mathrm{wt} \%$ PPG), after Reference 11 ; +, nylon-6/EPDM blends with various rubber contents $(2,5,8,10,20 \mathrm{wt} \%)$, average rubber particle size $>0.5 \mu \mathrm{m}$, after Reference $4 ; \triangle$, nylon-6/EPDM blends with various rubber contents $(2,5,8,10,20 \mathrm{wt} \%)$, average rubber particle size $\approx 0.3 \mu \mathrm{m}$, after Reference 4

rubber particle size, however, possess the combination of high toughness and high stiffness; these blends are very tough at rubber concentrations $>8 \mathrm{wt} \%$, even when the macroscopic yield stress of the material is high. Toughening mechanism 2 applies in this case.

According to Figure 5, below the nylon glass transition temperature cop 10 and cop20 have yield stresses above the critical level and consequently low notched Izod impact energies.

Above $-10^{\circ} \mathrm{C}$ cop30 has a $\sigma_{y}$ low enough to ensure a large amount of plastic deformation and therefore a large quantity of energy absorption during impact. Below $-10^{\circ} \mathrm{C} \sigma_{y}$ approaches $\sigma_{y \text {-crit }}$, which results in a decrease of the impact strength with decreasing temperature. However, the high toughness of cop30 is inevitably coupled with a low modulus of the material.

\section{ACKNOWLEDGEMENTS}

This work is part of the research programme of the University of Twente and was financially supported by the Netherlands Technology Foundation (STW) and by the DSM Research Company. The authors wish to thank Dr E. Roerdink (DSM) for stimulating discussions, Dr P. F. van Hutten, W. Busing (DSM) and B. Klazema (GEP) for their contributions in the electron microscope work and Professor J. Schuijer for his active interest.

\section{REFERENCES}

1 Ogorkiewicz, R. M. 'Engineering Properties of Plastics', Wiley, New York, 1970

2 Nelson, W. E. 'Nylon Plastics Technology', NewnesButterworths, Guildford, 1976

3 Gaymans, R. J., Borggreve, R. J. M. and Spoelstra, A. B. J. Appl. Polym. Sci. submitted

4 Borggreve, R. J. M., Gaymans, R. J., Schuijer, J. and Ingen Housz, J. F. Polymer 1987, 28, 1489

5 Flexman, E. A. Polym. Eng. Sci. 1979, 19, 564

Wu, S. Polymer 1985, 26, 1885

Wu, S. J. Polym. Sci., Polym. Phys. Edn 1983, 21, 699

Hobbs, S. Y., Bopp, R. C. and Watkins, V. H. Polym. Eng. Sci. 1983, 23, 1983

9 Brown, N. 'Failure of plastics', Hanser Publishers, Munich, 1986, p. 98

Illing, G. Angew. Makromol. Chem. 1981, 95, 83

11 Van der Loos, J. L. M. and Van Geenen, A. A. 'Symposium on New Polymeric Materials', Naples, 1986

12 Takayanagi, M. Mem. Fac. Eng. Kyushu Univ. 1963, 23, 57

13 Uemara, S. and Takayanagi, M. J. Appl. Polym. Sci. 1966, 10, 113

Van der Poel, C. Rheol. Acta 1958, 1, 198

Kerner, E. H. Proc. Phys. Soc. 1956, 69B, 808

Dickie, R. A. J. Appl. Polym. Sci. 1973, 17, 45

Bohn, L. Adv. Chem. Ser. 1975, 42, 66

Greco, R., Lanzetta, G., Maglio, G., Malinconico, M., Martuscelli, M., Palumbo, R., Ragosta, G. and Scarinzi, G. Polymer 1986, 27, 299

19 Wunderlich, B. 'Macromolecular Physics', vol. 3, Academic Press, New York, 1980

20 Bessel, T. J., Hull, D. and Shortall, J. B. J. Mater. Sci. 1975, 10, 1127

21 Ptaszinsky, B., Terisse, J. and Skoulios, A. Makromol. Chem. $1975,176,3483$

22 Schmitt, B. J. Angew. Chem. 1979, 91, 286

23 Borggreve, R. J. M., Gaymans, R. J. and Luttmer, A. R. Makromol. Chem. Makromol. Symp. 1988, 16, 195 